PIOTR JAWORSKI (Warszawa)

\title{
ON UNIFORM TAIL EXPANSIONS OF BIVARIATE COPULAS
}

Abstract. The theory of copulas provides a useful tool for modelling dependence in risk management. The goal of this paper is to describe the tail behaviour of bivariate copulas and its role in modelling extreme events. We say that a bivariate copula has a uniform lower tail expansion if near the origin it can be approximated by a homogeneous function $L(u, v)$ of degree 1 ; and it is said to have a uniform upper tail expansion if the associated survival copula has a lower tail expansion. In this paper we (1) introduce the notion of the uniform tail expansion of a bivariate copula; (2) describe the main properties of the leading part $L(u, v)$ like two-monotonicity or concavity; (3) determine the set of all possible leading parts $L(u, v)$; (4) compute the leading parts of the uniform tail expansions for the most popular copulas like gaussian, archimedean or BEV; (5) apply uniform tail expansions in estimating the extreme risk of a portfolio consisting of long positions in risky assets.

1. Introduction. The theory of copulas provides a useful tool for modelling dependence in risk management. The goal of this paper is to describe the tail behaviour of bivariate copulas and its role in modelling extreme events. We discuss possible applications, taking as an example a portfolio consisting of two risky assets.

We recall that a function $C:[0,1]^{2} \rightarrow[0,1]$ is called a copula (see [6]) if

$$
\begin{gathered}
\forall u, v \in[0,1] \quad C(0, v)=0, \quad C(u, 0)=0 ; \\
\forall u, v \in[0,1] \quad C(1, v)=v, \quad C(u, 1)=u ; \\
\forall u_{1}, u_{2}, v_{1}, v_{2} \in[0,1], u_{1} \leq u_{2}, v_{1} \leq v_{2}, \\
C\left(u_{1}, v_{2}\right)+C\left(u_{2}, v_{1}\right) \leq C\left(u_{1}, v_{1}\right)+C\left(u_{2}, v_{2}\right) .
\end{gathered}
$$

2000 Mathematics Subject Classification: 62H20, 62E20, 91B28, 91B30.

Key words and phrases: copulas, fat tails, dependence of extreme events, risk management, portfolio theory.

Partially supported by PBZ-KBN-016/P03/99. 
The functions with the last property are called two-nondecreasing. Every copula is nondecreasing with respect to each variable, continuous (and even Lipschitz).

Let $\mathcal{X}$ and $\mathcal{Y}$ be two random variables defined on the same probability space $(\Omega, \mathcal{M}, \mathbb{P})$. Their joint cumulative distribution $F_{\mathcal{X Y}}$ can be described using an appropriate bivariate copula $C_{\mathcal{X Y}}$ (see [6, Th. 2.4.1]):

$$
F_{\mathcal{X Y}}(x, y)=C_{\mathcal{X Y}}\left(F_{\mathcal{X}}(x), F_{\mathcal{Y}}(y)\right)
$$

where $F_{\mathcal{X}}, F_{\mathcal{Y}}$ are the cumulative distributions of $\mathcal{X}$ and $\mathcal{Y}$. Note that strictly increasing transformations of the random variables $\mathcal{X}$ and $\mathcal{Y}$ do not affect the copula. Indeed, if

$$
\mathcal{X}^{\prime}=f(\mathcal{X}), \quad \mathcal{Y}^{\prime}=g(\mathcal{Y}),
$$

where $f$ and $g$ are strictly increasing (and so invertible), then

$$
\begin{aligned}
F_{\mathcal{X}^{\prime} \mathcal{Y}^{\prime}}(x, y) & =F_{\mathcal{X Y Y}}\left(f^{-1}(x), g^{-1}(y)\right)=C\left(F_{\mathcal{X}}\left(f^{-1}(x)\right), F_{\mathcal{Y}}\left(g^{-1}(y)\right)\right) \\
& =C\left(F_{\mathcal{X}^{\prime}}(x), F_{\mathcal{Y}^{\prime}}(y)\right) .
\end{aligned}
$$

Therefore if one is interested in tail dependence of random variables rather than in their individual distribution, then the proper choice is to study the copula, the more so since the copula is uniquely determined at every point $(u, v)$ such that the equations $F_{\mathcal{X}}(x)=u$ and $F_{\mathcal{Y}}(y)=v$ have solutions.

For every copula $C$ we have a dual, so-called survival copula $\widehat{C}$, defined by

$$
\widehat{C}(u, v)=C(1-u, 1-v)+u+v-1 .
$$

Note that if $C$ describes the joint distribution of $\mathcal{X}$ and $\mathcal{Y}$, then $\widehat{C}$ does the same for $-\mathcal{X}$ and $-\mathcal{Y}$.

Furthermore, in certain cases the copula $C_{\mathcal{X Y}}$ is the joint cumulative distribution of some random variables defined on the same probability space as $\mathcal{X}$ and $\mathcal{Y}$. Indeed, let $\mathcal{P}$ and $\mathcal{Q}$ be the random variables defined by

$$
\mathcal{P}=F_{\mathcal{X}}(\mathcal{X}), \quad \mathcal{Q}=F_{\mathcal{Y}}(\mathcal{Y}) .
$$

Proposition 1. If the cumulative distributions $F_{\mathcal{X}}, F_{\mathcal{Y}}$ are continuous then:

(i) $\mathcal{P}$ and $\mathcal{Q}$ have uniform distributions on $[0,1]$.

(ii) The copula $C_{\mathcal{X Y}}$ is uniquely determined.

(iii) The two-dimensional cumulative distribution $F_{\mathcal{P} Q}$ coincides with the copula $C_{\mathcal{X Y}}$.

Proof. We prove (iii) (the first two items are obvious). We choose $p, q$ such that $0 \leq p, q \leq 1$. Since $F_{\mathcal{X}}$ and $F_{\mathcal{Y}}$ are continuous, there exist $x, y$ such that

$$
p=F_{\mathcal{X}}(x), \quad q=F_{\mathcal{Y}}(y)
$$


Hence

$$
\begin{aligned}
F_{\mathcal{P} Q}(p, q) & =\mathbb{P}(\mathcal{P} \leq p \wedge \mathcal{Q} \leq q)=\mathbb{P}\left(F_{\mathcal{X}}(\mathcal{X}) \leq F_{\mathcal{X}}(x) \wedge F_{\mathcal{Y}}(\mathcal{Y}) \leq F_{\mathcal{Y}}(y)\right) \\
& =\mathbb{P}(\mathcal{X} \leq x \wedge \mathcal{Y} \leq y)=C_{\mathcal{X} Y}\left(F_{\mathcal{X}}(x), F_{\mathcal{Y}}(y)\right)=C_{\mathcal{X Y Y}}(p, q)
\end{aligned}
$$

In order to study extreme events we have to deal with the tail behaviour of a copula. We say that a bivariate copula has a uniform lower tail expansion if near the origin it can be uniformly approximated by a homogeneous function of degree 1 . In more detail:

Definition. We say that a copula $C:[0,1]^{2} \rightarrow[0,1]$ has a lower tail expansion if there exists a homogeneous function $L: \mathbb{R}_{+}^{2} \rightarrow \mathbb{R}$ of degree 1 , i.e.

$$
\forall t \geq 0 \quad L(t u, t v)=t L(u, v)
$$

and a bounded function $R:[0,1]^{2} \rightarrow \mathbb{R}$ with

$$
\lim _{(u, v) \rightarrow(0,0)} R(u, v)=0
$$

such that

$$
\forall u, v \in[0,1] \quad C(u, v)=L(u, v)+R(u, v)(u+v) .
$$

Furthermore, we say that $C$ has a uniform upper tail expansion if the associated survival copula $\widehat{C}$ has a lower tail expansion.

The function $L$ will be called the leading part of the expansion. When $L \equiv 0$ we shall say that the expansion is trivial.

Our conditions are a bit stronger than the ones introduced by P. Embrechts ([1]) and other authors, but still they are satisfied by nearly all copulas studied in the literature.

There are also other ways of introducing the leading part $L$. For example one may adopt the definition with the "strong" derivative.

Lemma 1. A copula $C:[0,1]^{2} \rightarrow[0,1]$ has a lower tail expansion iff there exists a homogeneous function $L: \mathbb{R}_{+}^{2} \rightarrow \mathbb{R}$ of degree 1 such that

$$
\lim _{(u, v) \rightarrow(0,0)^{+}} \frac{|C(u, v)-C(0,0)-L(u, v)|}{\|(u, v)\|}=0 .
$$

Furthermore the value of $L$ can be determined as a limit along a ray.

LEMma 2.

$$
L(u, v)=\lim _{t \rightarrow 0^{+}} \frac{C(t u, t v)}{t} \quad \text { for } u, v \geq 0 .
$$

Proof. We have

$$
\begin{aligned}
& \frac{C(t u, t v)}{t}=\frac{L(t u, t v)+R(t u, t v)(t u+t v)}{t} \\
& \quad=\frac{t L(u, v)+t R(t u, t v)(u+v)}{t}=L(u, v)+R(t u, t v)(u+v) \rightarrow L(u, v) .
\end{aligned}
$$


In the following parts of the paper we:

- describe the main properties of uniform tail expansions and determine the set of all possible leading parts $L(u, v)$;

- compute the leading parts of the uniform tail expansions for the most popular copulas like gaussian, archimedean or BEV;

- apply uniform tail expansions in estimating the extreme risk of a portfolio consisting of long positions in risky assets.

2. Properties of the leading part $L$. Let $L(u, v)$ be a leading part of the uniform lower expansion of a given copula $C(u, v)$.

THEOREM 1. $L(u, v)$ is nonnegative, two-nondecreasing and bounded by the smaller of its arguments:

$$
\begin{gathered}
0 \leq L(u, v) \leq \min (u, v) \\
u_{1} \leq u_{2}, v_{1} \leq v_{2} \Rightarrow L\left(u_{1}, v_{2}\right)+L\left(u_{2}, v_{1}\right) \leq L\left(u_{1}, v_{1}\right)+L\left(u_{2}, v_{2}\right) .
\end{gathered}
$$

This follows directly from Lemma 2 (cf. [4, Th. 1]).

From the above theorem and the homogeneity of $L$ we obtain its further properties:

Corollary 1. Let $u, u_{1}, u_{2}, v, v_{1}, v_{2} \geq 0$. Then:

(i) $L(0, v)=L(u, 0)=0$.

(ii) If $u_{1} \leq u_{2}$ then $0 \leq L\left(u_{2}, v\right)-L\left(u_{1}, v\right) \leq u_{2}-u_{1}$ and if $v_{1} \leq v_{2}$ then $0 \leq L\left(u, v_{2}\right)-L\left(u, v_{1}\right) \leq v_{2}-v_{1}$.

(iii) $L(u, v)$ is continuous.

(iv) The function

$$
l:[0,1] \rightarrow \mathbb{R}, \quad l(\alpha)=L(\alpha, 1-\alpha),
$$

is concave and continuous.

(v) $L(u, v)$ is superadditive:

$$
L\left(u_{1}+u_{2}, v_{1}+v_{2}\right) \geq L\left(u_{1}, v_{1}\right)+L\left(u_{2}, v_{2}\right) .
$$

(vi) $L(u, v)$ is concave:

$$
\begin{aligned}
\forall \lambda_{1}, \lambda_{2} \geq & 0, \lambda_{1}+\lambda_{2}=1 \\
& L\left(\lambda_{1} u_{1}+\lambda_{2} u_{2}, \lambda_{1} v_{1}+\lambda_{2} v_{2}\right) \geq \lambda_{1} L\left(u_{1}, v_{1}\right)+\lambda_{2} L\left(u_{2}, v_{2}\right) .
\end{aligned}
$$

Proof. The first three properties are straightforward (see [4, Cor. 1]).

(iv) Since $L$ is continuous, so is $l(\alpha)=L(\alpha, 1-\alpha)$. The concavity of $l$ follows from the fact that $L$ is two-nondecreasing. Indeed, let $0<\alpha_{1}<$ $\alpha_{2}<1$. Since $1-\alpha_{2}<1-\alpha_{1}$, we have

$$
L\left(\alpha_{1}, 1-\alpha_{2}\right)+L\left(\alpha_{2}, 1-\alpha_{1}\right) \geq L\left(\alpha_{1}, 1-\alpha_{1}\right)+L\left(\alpha_{2}, 1-\alpha_{2}\right) .
$$


Let

$\alpha_{3}=\frac{\alpha_{1}}{1-\alpha_{2}+\alpha_{1}}, \alpha_{4}=\frac{\alpha_{2}}{1-\alpha_{1}+\alpha_{2}}, \varrho_{3}=1-\alpha_{2}+\alpha_{1}, \varrho_{4}=1-\alpha_{1}+\alpha_{2}$.

Then the above inequality can be written as

$$
\varrho_{3} l\left(\alpha_{3}\right)+\varrho_{4} l\left(\alpha_{4}\right) \geq l\left(\alpha_{1}\right)+l\left(\alpha_{2}\right) .
$$

Here

$$
\varrho_{3}+\varrho_{4}=2, \quad \varrho_{3} \alpha_{3}+\varrho_{4} \alpha_{4}=\alpha_{1}+\alpha_{2} .
$$

Moreover $\alpha_{3}$ and $\alpha_{4}$ lie between $\alpha_{1}$ and $\alpha_{2}$. Thus at least one of the points $\left(\alpha_{3}, l\left(\alpha_{3}\right)\right),\left(\alpha_{4}, l\left(\alpha_{4}\right)\right)$ lies above or on the chord joining $\left(\alpha_{1}, l\left(\alpha_{1}\right)\right)$ and $\left(\alpha_{2}, l\left(\alpha_{2}\right)\right)$.

We shall show that for any $-1<\beta_{1}<\beta_{2}<1$ the graph of $l$ lies above or on the chord joining $\left(\beta_{1}, l\left(\beta_{1}\right)\right)$ and $\left(\beta_{2}, l\left(\beta_{2}\right)\right)$. Let $Z \subset\left[\beta_{1}, \beta_{2}\right]$ consist of those $\beta$ such that $(\beta, l(\beta))$ lies over the chord. Then $Z$ is closed (because $l$ is continuous), nonempty (because $\beta_{1}$ and $\beta_{2}$ belong to it) and dense (because between any two points from $Z$ there is a third one), therefore $Z$ is the whole interval $\left[\beta_{1}, \beta_{2}\right]$. Since $l$ is continuous, this finishes the proof of the concavity for the whole domain $[0,1]$.

(v) Assume that $u_{1}+v_{1}>0$ and $u_{2}+v_{2}>0$. Let

$$
\alpha_{1}=\frac{u_{1}}{v_{1}+u_{1}}, \quad \alpha_{2}=\frac{u_{2}}{v_{2}+u_{2}} \text {. }
$$

Then

$$
L\left(u_{i}, v_{i}\right)=\left(u_{i}+v_{i}\right) l\left(\alpha_{i}\right) \quad \text { for } i=1,2,
$$

and

$$
\begin{aligned}
& L\left(u_{1}+u_{2}, v_{1}+v_{2}\right) \\
& =\left(u_{1}+u_{2}+v_{1}+v_{2}\right) l\left(\frac{u_{1}+v_{1}}{u_{1}+u_{2}+v_{1}+v_{2}} \alpha_{1}+\frac{u_{2}+v_{2}}{u_{1}+u_{2}+v_{1}+v_{2}} \alpha_{2}\right) \\
& \geq\left(u_{1}+u_{2}+v_{1}+v_{2}\right)\left(\frac{u_{1}+v_{1}}{u_{1}+u_{2}+v_{1}+v_{2}} l\left(\alpha_{1}\right)+\frac{u_{2}+v_{2}}{u_{1}+u_{2}+v_{1}+v_{2}} l\left(\alpha_{2}\right)\right) \\
& =\left(u_{1}+v_{1}\right) l\left(\alpha_{1}\right)+\left(u_{2}+v_{2}\right) l\left(\alpha_{2}\right)=L\left(u_{1}, v_{1}\right)+L\left(u_{2}, v_{2}\right) .
\end{aligned}
$$

(vi) is a direct consequence of (v). Indeed, let $\lambda_{1}, \lambda_{2} \geq 0$ and $\lambda_{1}+\lambda_{2}=1$. Then

$$
\begin{aligned}
L\left(\lambda_{1} u_{1}+\lambda_{2} u_{2}, \lambda_{1} v_{1}+\lambda_{2} v_{2}\right) & \geq L\left(\lambda_{1} u_{1}, \lambda_{1} v_{1}\right)+L\left(\lambda_{2} u_{2}, \lambda_{2} v_{2}\right) \\
& =\lambda_{1} L\left(u_{1}, v_{1}\right)+\lambda_{2} L\left(u_{2}, v_{2}\right) .
\end{aligned}
$$

For another proof of concavity of $L$, based on its differential properties, the reader is referred to [4, Th. 2]. As a matter of fact for homogeneous functions of degree 1 the concavity and two-monotonicity are closely related. 
Proposition 2. Let $L: \mathbb{R}_{+}^{2} \rightarrow \mathbb{R}$ be homogeneous of degree 1 , concave and continuous. Then $L$ is two-nondecreasing.

Proof. Let $0 \leq v_{1} \leq v_{2}$ and $0 \leq u_{1} \leq u_{2}$. We have to show that

$$
L\left(u_{1}, v_{1}\right)+L\left(u_{2}, v_{2}\right)-L\left(u_{1}, v_{2}\right)-L\left(u_{2}, v_{1}\right) \geq 0 .
$$

Since $L$ is continuous, it is enough to consider the case $u_{1}>0$. Note that since $L$ is concave and continuous, the same is true for

$$
l:[0,1] \rightarrow \mathbb{R}, \quad l(\alpha)=L(\alpha, 1-\alpha) .
$$

Since $L$ is homogeneous of degree 1 , we have

$$
\begin{aligned}
& L\left(u_{1}, v_{1}\right)+L\left(u_{2}, v_{2}\right)-L\left(u_{1}, v_{2}\right)-L\left(u_{2}, v_{1}\right) \\
& =\left(u_{1}+v_{1}\right) l\left(\frac{u_{1}}{u_{1}+v_{1}}\right)+\left(u_{2}+v_{2}\right) l\left(\frac{u_{2}}{u_{2}+v_{2}}\right) \\
& \quad-\left(u_{1}+v_{2}\right) l\left(\frac{u_{1}}{u_{1}+v_{2}}\right)-\left(u_{2}+v_{1}\right) l\left(\frac{u_{2}}{u_{2}+v_{1}}\right) \\
& =\left(u_{1}+u_{2}+v_{1}+v_{2}\right)\left(l_{1}\left(\frac{u_{1}+u_{2}}{u_{1}+u_{2}+v_{1}+v_{2}}\right)-l_{2}\left(\frac{u_{1}+u_{2}}{u_{1}+u_{2}+v_{1}+v_{2}}\right)\right) .
\end{aligned}
$$

Here $l_{1}$ and $l_{2}$ are linear functions whose graphs contain chords joining points on the graph of $l$ :

$$
\begin{array}{ll}
l_{1}\left(\frac{u_{1}}{u_{1}+v_{1}}\right)=l\left(\frac{u_{1}}{u_{1}+v_{1}}\right), & l_{1}\left(\frac{u_{2}}{u_{2}+v_{2}}\right)=l\left(\frac{u_{2}}{u_{2}+v_{2}}\right), \\
l_{2}\left(\frac{u_{1}}{u_{1}+v_{2}}\right)=l\left(\frac{u_{1}}{u_{1}+v_{2}}\right), & l_{2}\left(\frac{u_{2}}{u_{2}+v_{1}}\right)=l\left(\frac{u_{2}}{u_{2}+v_{1}}\right) .
\end{array}
$$

Note that since $v_{1} \leq v_{2}$ and $0<u_{1} \leq u_{2}$, we have

$$
\begin{aligned}
& \frac{u_{1}}{u_{1}+v_{2}} \leq \frac{u_{1}}{u_{1}+v_{1}}=\frac{1}{1+v_{1} / u_{1}} \leq \frac{1}{1+v_{1} / u_{2}}=\frac{u_{2}}{u_{2}+v_{1}} \\
& \frac{u_{1}}{u_{1}+v_{2}}=\frac{1}{1+v_{2} / u_{1}} \leq \frac{1}{1+v_{2} / u_{2}}=\frac{u_{2}}{u_{2}+v_{2}} \leq \frac{u_{2}}{u_{2}+v_{1}}
\end{aligned}
$$

Therefore the first chord lies above the second one and

$$
\left(u_{1}+u_{2}+v_{1}+v_{2}\right)\left(l_{1}\left(\frac{u_{1}+u_{2}}{u_{1}+u_{2}+v_{1}+v_{2}}\right)-l_{2}\left(\frac{u_{1}+u_{2}}{u_{1}+u_{2}+v_{1}+v_{2}}\right)\right) \geq 0
$$

which finishes the proof.

2.1. Copulas with prescribed leading part $L$. In this section we shall show that Theorem 1 completely describes all possible leading parts.

TheOREM 2. Let $L: \mathbb{R}_{+}^{2} \rightarrow \mathbb{R}$ be homogeneous of degree 1 , two-nondecreasing and such that

$$
\forall u, v \quad 0 \leq L(u, v) \leq \min (u, v) .
$$


Then the function

$$
C(u, v)=\max (L(u, v), u+v-1)
$$

restricted to the unit square $[0,1]^{2}$ is a copula.

The proof is based on checking all possibilities (compare [4, Th. 3]).

Note that for any $L$ which is nonnegative, and for $0 \leq u+v \leq 1$,

$$
C(u, v)=\max (L(u, v), u+v-1)=L(u, v) .
$$

Thus $L$ is not only the lower tail approximation of $C$ but the tail itself.

The above theorem together with Proposition 2 and Corollary 1 gives us the following:

Corollary 2. There is a bijection between

- leading parts $L$ of uniform tail expansions and

- concave functions $l:[0,1] \rightarrow \mathbb{R}$ which satisfy

$$
0 \leq l(\alpha) \leq \min (\alpha, 1-\alpha) .
$$

The bijection is given by the formula

$$
l(\alpha)=L(\alpha, 1-\alpha) .
$$

The above results remain valid for pairs of tails and pairs of functions.

Theorem 3. Any two two-nondecreasing functions $L_{i}: \mathbb{R}_{+}^{2} \rightarrow \mathbb{R}, i=$ 1,2 , homogeneous of degree 1 and such that

$$
0 \leq L_{i}(u, v) \leq \min (u, v),
$$

are the leading parts of the lower and upper tail expansions of some copula.

Proof. We shall apply the patchwork technique (cf. [6]). For $i=1,2$ let

$$
C_{i}(u, v)=\max \left(L_{i}(u, v), u+v-1\right) .
$$

Then the function $C:[0,1]^{2} \rightarrow[0,1]$ defined by

$$
C(u, v)= \begin{cases}C_{1}(2 u, 2 v) / 2 & \text { for } 0 \leq u, v \leq 1 / 2, \\ u & \text { for } 0 \leq u \leq 1 / 2<v, \\ v & \text { for } 0 \leq v \leq 1 / 2<u, \\ \left(2 u+2 v-2+C_{2}(2-2 u, 2-2 v)\right) / 2 & \text { for } 1 / 2<u, v \leq 1,\end{cases}
$$

is a copula. Indeed, for the lower left corner we apply the measure induced by $C_{1}$, for the upper right one the measure induced by $\widehat{C}_{2}$, and for the remaining two the null measure. 


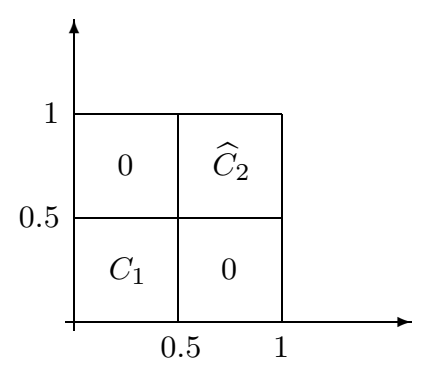

Therefore for $u+v<1 / 2$,

$$
C(u, v)=\frac{C_{1}(2 u, 2 v)}{2}=L_{1}(u, v), \quad \widehat{C}(u, v)=\frac{C_{2}(2 u, 2 v)}{2}=L_{2}(u, v) .
$$

3. Examples of trivial expansions. Assume that $\mathcal{X}$ and $\mathcal{Y}$ are independent. Then

$$
C(u, v)=u v .
$$

In this case $L(u, v)=0$ and $R(u, v)=u v /(u+v)$. Hence the expansion of the lower tail is trivial. The same is valid for the survival copula. Hence the expansion of the upper tail is also trivial.

Let now $\mathcal{X}$ and $\mathcal{Y}$ have the same standard normal distribution $N(0,1)$ and normal joint distribution. Then their copula

$$
C_{N}(u, v)=F_{\mathcal{X Y}}\left(F^{-1}(u), F^{-1}(v)\right),
$$

where $F$ is the distribution function of the standard normal distribution $(N(0,1))$, is called gaussian.

Proposition 3. The gaussian copula $C_{N}$ has trivial expansions of both tails.

The proof of the lower tail case (see [4, Lemma 1]) is based on the fact that the shifted quadrant $\left(-\infty, x_{0}\right] \times\left(-\infty, y_{0}\right]$ is contained in the halfplane $\left\{(x, y): x+y \leq x_{0}+y_{0}\right\}$ and on an estimation of the tail of the standard normal distribution (see [3, p. 119]). The upper tail case is quite similar.

REMARK 1. The above result shows that the phenomenon of nontrivial tail expansions does not exist in the "world ruled by the paradigm of normality of all distributions".

4. Simple examples of nontrivial expansions. Assume that $\mathcal{Y}=\mathcal{X}$. Then

$$
C(u, v)=\min (u, v) .
$$

In this case $L(u, v)=\min (u, v)$ and $R(u, v)=0$. The same is true for the survival copula. Note that the above remains true if we only assume that $\mathcal{Y}$ and $\mathcal{X}$ are comonotonic. 
The above copula has a singular support (the diagonal $\{(t, t): t \in[0,1]\})$. Other examples of singular support copulas are given by

$$
C_{p q}(u, v)=\max ((\varrho-1) \min (u / p, v / q), u+v-1),
$$

where $p+q=\varrho>1,0<p, q \leq 1$. The support consists of one, two or three segments, each with uniform distribution of mass.

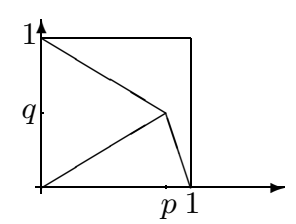

Except the case $p=q=1$ the upper tail has a trivial expansion. But the lower tail always has a nonzero leading part:

$$
\begin{aligned}
L(u, v) & =(\varrho-1) \min (u / p, v / q), & & =p+q, \\
l(\varphi) & =\left(1-\frac{1}{\varrho}\right) \min \left(\frac{\varphi}{\psi}, \frac{1-\varphi}{1-\psi}\right), & \psi & =\frac{p}{\varrho} .
\end{aligned}
$$

\section{Archimedean and quasi-archimedean copulas}

5.1. Definitions. Let $\varphi:[0,1] \rightarrow[0, \infty]$ be a convex, strictly decreasing continuous function with $\varphi(1)=0$ and $\varphi(0)=\varphi_{0} \leq \infty$. Then the function

$$
C:[0,1]^{2} \rightarrow[0,1], \quad C(u, v)=\varphi^{-1}\left(\min \left(\varphi_{0}, \varphi(u)+\varphi(v)\right)\right),
$$

is a copula (see [6, Th. 4.1.4]). Such copulas are called archimedean.

We extend this notion by setting

$$
C_{\varphi, H}(u, v)=\varphi^{-1}\left(\min \left(\varphi_{0}, \varphi(u)+\varphi(v)-H(\varphi(u), \varphi(v))\right)\right),
$$

where $H: \mathbb{R}_{+}^{2} \rightarrow \mathbb{R}$ is homogeneous of degree 1 , two-nondecreasing and such that

$$
\forall u, v \quad 0 \leq H(u, v) \leq \min (u, v) .
$$

Theorem 4. $C_{\varphi, H}$ is a copula.

Proof. We have

$$
H(\varphi(u), \varphi(v)) \leq \min (\varphi(u), \varphi(v)) \leq \varphi(u)+\varphi(v) .
$$

Hence $C_{\varphi, H}$ is well defined. Moreover

$$
\begin{aligned}
C_{\varphi, H}(0, v) & =\varphi^{-1}\left(\min \left(\varphi_{0}, \varphi(0)+\varphi(v)-H(\varphi(0), \varphi(v))\right)\right) \\
& =\varphi^{-1}\left(\min \left(\varphi_{0}, \varphi_{0}+\varphi(v)-H\left(\varphi_{0}, \varphi(v)\right)\right)\right)=\varphi^{-1}\left(\varphi_{0}\right)=0, \\
C_{\varphi, H}(1, v) & =\varphi^{-1}\left(\min \left(\varphi_{0}, \varphi(1)+\varphi(v)-H(\varphi(1), \varphi(v))\right)\right) \\
& =\varphi^{-1}\left(\min \left(\varphi_{0}, 0+\varphi(v)-H(0, \varphi(v))\right)\right)=\varphi^{-1}\left(\min \left(\varphi_{0}, \varphi(v)\right)\right) \\
& =\varphi^{-1}(\varphi(v))=v .
\end{aligned}
$$

Analogously, $C_{\varphi, H}(u, 0)=0$ and $C_{\varphi, H}(u, 1)=u$. 
Next we show that $C_{\varphi, H}$ is two-nondecreasing. Let $u_{1} \leq u_{2}$ and $v_{1} \leq v_{2}$. We put

$$
\begin{aligned}
& x_{1}=\varphi\left(u_{1}\right)+\varphi\left(v_{1}\right)-H\left(\varphi\left(u_{1}\right), \varphi\left(v_{1}\right)\right), \\
& x_{2}=\varphi\left(u_{1}\right)+\varphi\left(v_{2}\right)-H\left(\varphi\left(u_{1}\right), \varphi\left(v_{2}\right)\right), \\
& x_{3}=\varphi\left(u_{2}\right)+\varphi\left(v_{1}\right)-H\left(\varphi\left(u_{2}\right), \varphi\left(v_{1}\right)\right), \\
& x_{4}=\varphi\left(u_{2}\right)+\varphi\left(v_{2}\right)-H\left(\varphi\left(u_{2}\right), \varphi\left(v_{2}\right)\right) .
\end{aligned}
$$

Since $\varphi$ is decreasing and $H$ satisfies the Lipschitz condition (see Corollary 1), we have

$$
x_{1} \geq x_{2} \geq x_{4}, \quad x_{1} \geq x_{3} \geq x_{4} .
$$

Since $H$ is two-nondecreasing, we have

$$
x_{1}-x_{2} \leq x_{3}-x_{4} .
$$

The function $\psi(x)=\varphi^{-1}\left(\min \left(\varphi_{0}, x\right)\right)$ is convex nonincreasing. The interval $\left[x_{2}, x_{1}\right]$ is shorter than $\left[x_{4}, x_{3}\right]$ and lies to the right of it, therefore

$$
\psi\left(x_{4}\right)-\psi\left(x_{3}\right) \geq \psi\left(x_{2}\right)-\psi\left(x_{1}\right) .
$$

Hence

$$
\begin{aligned}
C_{\varphi, H}\left(u_{2}, v_{2}\right)-C_{\varphi, H}\left(u_{2}, v_{1}\right) & =\psi\left(x_{4}\right)-\psi\left(x_{3}\right) \geq \psi\left(x_{2}\right)-\psi\left(x_{1}\right) \\
& =C_{\varphi, H}\left(u_{1}, v_{2}\right)-C_{\varphi, H}\left(u_{1}, v_{1}\right)
\end{aligned}
$$

which finishes the proof.

We call the copula $C_{\varphi, H}$ quasi-archimedean. Note that the class of quasiarchimedean copulas contains among others the bivariate extreme value (BEV) copulas investigated for example in [2].

REMARK 2. If $H(u, v)=\min (u, v)$ then for any $\varphi$,

$$
\begin{aligned}
& C_{\varphi, H}(u, v)=\varphi^{-1}\left(\min \left(\varphi_{0}, \varphi(u)+\varphi(v)-\min (\varphi(u), \varphi(v))\right)\right) \\
& \quad=\varphi^{-1}\left(\min \left(\varphi_{0}, \max (\varphi(u), \varphi(v))\right)\right)=\varphi^{-1}(\max (\varphi(u), \varphi(v)))=\min (u, v) .
\end{aligned}
$$

Hence the tail expansions do not depend on $\varphi$. Both leading parts are equal to $\min (u, v)$.

5.2. Elasticity. We recall that the elasticity of a differentiable positive real-valued function $f$ is defined by

$$
\mathcal{E}_{x} f(x)=\frac{x f^{\prime}(x)}{f(x)} .
$$

LEMma 3. Let $f:(0, \varepsilon) \rightarrow(0, \infty)$ be differentiable with elasticity bounded by $d_{0}$ and $d_{1}$ :

$$
\forall x \in(0, \varepsilon) \quad d_{0} \leq \mathcal{E}_{x} f(x) \leq d_{1} .
$$

If $d_{0} \neq-\infty$ then

$$
\forall x \in(0, \varepsilon) \forall t \in(0,1] \quad t^{d_{0}} f(x) \geq f(t x) .
$$


If $d_{1} \neq \infty$ then

$$
\forall x \in(0, \varepsilon) \forall t \in(0,1] \quad t^{d_{1}} f(x) \leq f(t x) .
$$

Proof. The proof is based on the following observation. For a given constant $d$ we consider the composite function $F(t, x)=t^{-d} f(t x), 1 \geq t>0$, $\varepsilon>x>0$. Then

$$
\begin{aligned}
\frac{\partial \ln (F(t, x))}{\partial t} & =\frac{\partial}{\partial t}(-d \ln (t)+\ln (f(t x)))=-\frac{d}{t}+\frac{x f^{\prime}(t x)}{f(t x)} \\
& =\frac{1}{t}\left(\mathcal{E}_{x} f(t x)-d\right) .
\end{aligned}
$$

Hence if $d \geq \sup \left\{\mathcal{E}_{x} f(x)\right\}$ then $F$ is decreasing in $t$ and for $t<1$ we get

$$
f(t x) \geq t^{d} f(x) .
$$

Analogously, if $d \leq \inf \left\{\mathcal{E}_{x} f(x)\right\}$ then $F$ is increasing in $t$ and for $t \leq 1$ we get

$$
f(t x) \leq t^{d} f(x)
$$

5.3. Tail expansions of quasi-archimedean copulas. In this subsection we assume that $H(u, v)<\min (u, v)$ for some $u, v$. We shall show that then the lower (resp. upper) tail expansion of the quasi-archimedean copula depends on the limit elasticity of $\varphi(x)($ resp. $\varphi(1-x))$ at 0 ,

$$
\mathcal{E}_{x}(0)=\lim _{x \rightarrow 0^{+}} \frac{x \varphi^{\prime}(x)}{\varphi(x)}, \quad \mathcal{E}_{x}(1)=\lim _{x \rightarrow 0^{+}} \frac{-x \varphi^{\prime}(1-x)}{\varphi(1-x)} .
$$

The only exception concerns the lower tail expansion. Namely if $\varphi_{0}$ is finite then $C_{\varphi, H}(u, v)$ vanishes in some neighbourhood of the origin, so $L(u, v)=0$.

THEOREM 5. If the limit $\mathcal{E}_{x}(1)$ exists then the quasi-archimedean copula $C_{\varphi, H}$ has a uniform upper tail expansion. Moreover, if $\mathcal{E}_{x}(1)=d<\infty$, then

$$
L(u, v)=u+v-\sqrt[d]{u^{d}+v^{d}-H\left(u^{d}, v^{d}\right)},
$$

and if $\mathcal{E}_{x}(1)=\infty$, then

$$
L(u, v)=\min (u, v) .
$$

Proof. Note that since $\varphi(1-x)$ is increasing and convex, $d$ cannot be smaller than 1 . Hence for any $d_{0}$ and $d_{1}$ such that $0<d_{0}<d<d_{1}$, we can find $\varepsilon$ such that for $x \in(0, \varepsilon)$ and $s \in(0,1)$,

$$
\begin{aligned}
s^{d_{1}} \varphi(1-x) & <\varphi(1-s x)<s^{d_{0}} \varphi(1-x), \\
s^{1 / d_{0}}\left(1-\varphi^{-1}(x)\right) & <1-\varphi^{-1}(s x)<s^{1 / d_{1}}\left(1-\varphi^{-1}(x)\right) .
\end{aligned}
$$

Therefore we have the following upper bound for $t$ close to 0 and $u+v<1$ : 


$$
\begin{aligned}
\widehat{C}_{\varphi, H} & (t u, t v)=\varphi^{-1}(\varphi(1-t u)+\varphi(1-t v)-H(\varphi(1-t u), \varphi(1-t v)))+t u+t v-1 \\
& \leq \varphi^{-1}\left(u^{d_{1}} \varphi(1-t)+v^{d_{1}} \varphi(1-t)-H\left(u^{d_{0}}, v^{d_{0}}\right) \varphi(1-t)\right)+t u+t v-1 \\
& \leq \sqrt[d_{0}]{u^{d_{1}}+v^{d_{1}}-H\left(u^{d_{0}}, v^{d_{0}}\right)}\left(1-\varphi^{-1}(\varphi(1-t))\right)+t u+t v \\
& =t\left(u+v-\sqrt[d_{\rho}]{u^{d_{1}}+v^{d_{1}}-H\left(u^{d_{0}}, v^{d_{0}}\right)}\right) .
\end{aligned}
$$

Analogously we get the lower bound

$$
\widehat{C}_{\varphi, H}(t u, t v) \geq t\left(u+v-\sqrt[d_{1}]{u^{d_{0}}+v^{d_{0}}-H\left(u^{d_{1}}, v^{d_{1}}\right)}\right) .
$$

Thus we have

$$
\begin{aligned}
& u+v-\sqrt[d_{1}]{u^{d_{0}}+v^{d_{0}}-H\left(u^{d_{1}}, v^{d_{1}}\right)} \\
& \leq \frac{\widehat{C}_{\varphi, H}(t u, t v)}{t} \leq u+v-\sqrt[d_{\rho}]{u^{d_{1}}+v^{d_{1}}-H\left(u^{d_{0}}, v^{d_{0}}\right)} .
\end{aligned}
$$

Passing to the limit as $t \rightarrow 0$ and $d_{0}, d_{1} \rightarrow d$ we get

$$
L(u, v)=u+v-\sqrt[d]{u^{d}+v^{d}-H\left(u^{d}, v^{d}\right)} .
$$

If $\mathcal{E}_{x}(1)=\infty$ then for any $d_{0}>0$ we can find $\varepsilon<1$ such that for $x \in(0, \varepsilon)$,

$$
1-\varphi^{-1}(2 x)<\sqrt[d_{0}]{2}\left(1-\varphi^{-1}(x)\right)
$$

Therefore we have the following lower bound for $t$ close to 0 and $u+v<1$ :

$$
\begin{aligned}
\widehat{C}_{\varphi, H}(t u, t v)= & \varphi^{-1}(\varphi(1-t u)+\varphi(1-t v)-H(\varphi(1-t u), \varphi(1-t v))) \\
& +t u+t v-1 \\
\geq & \varphi^{-1}(2 \max (\varphi(1-t u), \varphi(1-t v)))+t u+t v-1 \\
\geq & -\sqrt[d_{0}]{2}(1-\min (1-t u, 1-t v))+t u+t v \\
= & t\left(u+v-\sqrt[d_{0}]{2} \max (u, v)\right) .
\end{aligned}
$$

The upper bound is obvious:

$$
\widehat{C}_{\varphi, H}(t u, t v) \leq \min (t u, t v)=t \min (u, v) .
$$

Thus we have

$$
u+v-\sqrt[d_{0}]{2} \max (u, v) \leq \frac{\widehat{C}_{\varphi, H}(t u, t v)}{t} \leq \min (u, v) .
$$

Passing to the limit as $d_{0} \rightarrow \infty$ we get $L(u, v)=\min (u, v)$.

THEOREM 6. If the limit $\mathcal{E}_{x}(0)$ exists then the quasi-archimedean copula $C_{\varphi, H}$ has a uniform lower tail expansion. Moreover if $\mathcal{E}_{x}(0)=-d, 0<d<\infty$ then

$$
L(u, v)=\frac{u v}{\sqrt[d]{u^{d}+v^{d}-H\left(u^{d}, v^{d}\right)}} ;
$$

if $\mathcal{E}_{x}(0)=-\infty$, then $L(u, v)=\min (u, v)$; and if $\mathcal{E}_{x}(0)=0$, then $L(u, v)=0$. 
Proof. Note that since $\varphi(x)$ is decreasing and convex, $-d$ cannot be positive. If $d$ is finite and nonzero then for any $d_{0}$ and $d_{1}$ such that $0<d_{0}<$ $d<d_{1}$, we can find $\varepsilon$ such that for $x \in(0, \varepsilon), y>\varepsilon^{-1}$ and $s \in(0,1)$,

$$
s^{-d_{0}} \varphi(x)<\varphi(s x)<s^{-d_{1}} \varphi(x), \quad s^{1 / d_{0}} \varphi^{-1}(y)<\varphi^{-1}\left(s^{-1} y\right)<s^{1 / d_{1}} \varphi^{-1}(y) .
$$

Therefore we have the following lower bound for $t$ close to 0 and $u+v<1$ :

$$
\begin{aligned}
C_{\varphi, H}(t u, t v) & =\varphi^{-1}(\varphi(t u)+\varphi(t v)-H(\varphi(t u), \varphi(t v))) \\
& \leq \varphi^{-1}\left(u^{-d_{0}} \varphi(t)+v^{-d_{0}} \varphi(t)-H\left(u^{-d_{1}}, v^{-d_{1}}\right) \varphi(t)\right) \\
& \leq \sqrt[-d_{1}]{u^{-d_{0}}+v^{-d_{0}}-H\left(u^{-d_{1}}, v^{-d_{1}}\right)} \varphi^{-1}(\varphi(t)) \\
& =t \sqrt[-d_{1}]{u^{-d_{0}}+v^{-d_{0}}-H\left(u^{-d_{1}}, v^{-d_{1}}\right)}
\end{aligned}
$$

Analogously we get the lower bound

$$
C_{\varphi, H}(t u, t v) \geq t \sqrt[-d_{0}]{u^{-d_{1}}+v^{-d_{1}}-H\left(u^{-d_{0}}, v^{-d_{0}}\right)} .
$$

Thus we have

$$
\begin{aligned}
& \sqrt[-d_{1}]{u^{-d_{0}+v^{-d_{0}}-H\left(u^{-d_{1}}, v^{-d_{1}}\right)}} \\
& \quad \geq \frac{C_{\varphi, H}(t u, t v)}{t} \geq \sqrt[-d_{0}]{u^{-d_{1}}+v^{-d_{1}}-H\left(u^{-d_{0}}, v^{-d_{0}}\right)} .
\end{aligned}
$$

Passing to the limit we get

$$
L(u, v)=\sqrt[-d]{u^{-d}+v^{-d}-H\left(u^{-d}, v^{-d}\right)}=\frac{u v}{\sqrt[d]{u^{d}+v^{d}-H\left(u^{d}, v^{d}\right)}} .
$$

If $\mathcal{E}_{x}(0)=-\infty$ then for any $d_{0}>0$ we can find $\varepsilon<1$ such that for $x \in(0, \varepsilon)$,

$$
\varphi^{-1}(2 x)>\sqrt[-d_{0}]{2} \varphi^{-1}(x)
$$

Therefore we have the following lower bound for $t$ close to 0 and $u+v<1$ :

$$
\begin{aligned}
C_{\varphi, H}(t u, t v) & =\varphi^{-1}(\varphi(t u)+\varphi(t v)-H(\varphi(t u), \varphi(t v))) \\
& \geq \varphi^{-1}(2 \max (\varphi(t u), \varphi(t v))) \\
& \geq \sqrt[-d_{0}]{2} \min (t u, t v)=t \sqrt[-d_{0}]{2} \min (u, v) .
\end{aligned}
$$

The upper bound is obvious:

$$
C_{\varphi, H}(t u, t v) \leq \min (t u, t v)=t \min (u, v) .
$$

Thus we have

$$
\sqrt[-d_{0}]{2} \min (u, v) \leq \frac{C_{\varphi, H}(t u, t v)}{t} \leq \min (u, v) .
$$

Passing to the limit as $d_{0} \rightarrow \infty$ we get $L(u, v)=\min (u, v)$.

If $\mathcal{E}_{x}(0)=0$ then for any $d_{1}>0$ we can find $\varepsilon$ such that for $x \in(0, \varepsilon)$, $y>\varepsilon^{-1}$ and $s \in(0,1)$,

$$
\varphi(x)<\varphi(s x)<s^{-d_{1}} \varphi(x), \quad \varphi^{-1}\left(s^{-1} y\right)<s^{1 / d_{1}} \varphi^{-1}(y) .
$$


Therefore we have the following upper bound for $t$ close to 0 and $u+v<1$ :

$$
\begin{aligned}
C_{\varphi, H}(t u, t v) & =\varphi^{-1}(\varphi(t u)+\varphi(t v)-H(\varphi(t u), \varphi(t v))) \\
& \leq \varphi^{-1}\left(\varphi(t)+\varphi(t)-H\left(\varphi(t) u^{-d_{1}}, \varphi(t) v^{-d_{1}}\right)\right) \\
& =\varphi^{-1}\left(\varphi(t)\left(2-H\left(u^{-d_{1}}, v^{-d_{1}}\right)\right)\right) \\
& \leq t \sqrt[-d_{1}]{2-H\left(u^{-d_{1}}, v^{-d_{1}}\right)} .
\end{aligned}
$$

Thus we have

$$
0 \leq \frac{C_{\varphi, H}(t u, t v)}{t} \leq \sqrt[-d_{1}]{2-H\left(u^{-d_{1}}, v^{-d_{1}}\right)}
$$

Passing to the limit as $d_{1} \rightarrow 0^{+}$we get $L(u, v)=0$.

\begin{tabular}{|c|c|c|c|}
\hline$\varphi(x)=\frac{1}{d}\left(x^{-d}-1\right)$ & $d \in[-1,0)$ & $\mathcal{E}_{x}(1)=1$ & $\varphi(0)<\infty$ \\
\hline$\varphi(x)=\frac{1}{d}\left(x^{-d}-1\right)$, & $d \in(0, \infty)$ & $\mathcal{E}_{x}(1)=1$ & $\mathcal{E}_{x}(0)=-d$ \\
\hline$\varphi(x)=(1-x)^{d}$ & $d \in[1, \infty)$ & $\mathcal{E}_{x}(1)=d$ & $\varphi(0)<\infty$ \\
\hline$\varphi(x)=\ln \frac{1-d(1-x)}{x}$, & $d \in[-1,1)$ & $\mathcal{E}_{x}(1)=1$, & $\mathcal{E}_{x}(0)=0$ \\
\hline$\varphi(x)=(-\ln x)^{d}$ & $d \in[1, \infty)$ & $\mathcal{E}_{x}(1)=d$ & $\mathcal{E}_{x}(0)=0$ \\
\hline$\varphi(x)=-\ln \frac{e^{-d x}-1}{e^{-d}-1}$ & $d \in(-\infty, \infty) \backslash\{0\}$ & $\mathcal{E}_{x}(1)=1$ & $\mathcal{E}_{x}(0)=0$ \\
\hline$\varphi(x)=-\ln \left(1-(1-x)^{d}\right)$ & $d \in[1, \infty)$ & $\mathcal{E}_{x}(1)=d$ & $\mathcal{E}_{x}(0)=0$ \\
\hline$\varphi(x)=-\ln (d x+1-d)$ & $d \in(0,1)$ & $\mathcal{E}_{x}(1)=1$, & $\varphi(0)<\infty$ \\
\hline$\varphi(x)=\frac{1-x}{1+(d-1) x}$ & $d \in[1, \infty)$ & $\mathcal{E}_{x}(1)=1$ & $\varphi(0)<\infty$ \\
\hline$\varphi(x)=\ln (1-d \ln (x))$ & $d \in(0,1]$ & $\mathcal{E}_{x}(1)=1$, & $\mathcal{E}_{x}(0)=0$ \\
\hline$\varphi(x)=\ln \left(2 x^{-d}-1\right)$ & $d \in(0,1]$ & $\mathcal{E}_{x}(1)=1$, & $\mathcal{E}_{x}(0)=0$ \\
\hline$\varphi(x)=\ln \left(2-x^{d}\right)$ & $d \in\left(0, \frac{1}{2}\right]$ & $\mathcal{E}_{x}(1)=1$, & $\varphi(0)<\infty$ \\
\hline$\varphi(x)=(1 / x-1)^{d}$ & $d \in[1, \infty)$ & $\mathcal{E}_{x}(1)=d$, & $\mathcal{E}_{x}(0)=-d$ \\
\hline$\varphi(x)=(1-\ln (x))^{d}-1$, & $d \in(0, \infty)$ & $\mathcal{E}_{x}(1)=1$ & $\mathcal{E}_{x}(0)=0$ \\
\hline$\varphi(x)=\left(x^{-1 / d}-1\right)^{d}$ & $d \in[1, \infty)$ & $\mathcal{E}_{x}(1)=d$, & $\mathcal{E}_{x}(0)=-d$ \\
\hline$\varphi(x)=\left(1-x^{1 / d}\right)^{d}$ & $d \in[1, \infty)$ & $\mathcal{E}_{x}(1)=d$ & $\varphi(0)<\infty$ \\
\hline$\varphi(x)=(d / x+1)(1-x)$, & $d \in(0, \infty)$ & $\mathcal{E}_{x}(1)=1$ & $\mathcal{E}_{x}(0)=-1$ \\
\hline$\varphi(x)=-\ln \frac{(1+x)^{-d}-1}{2^{-d}-1}$ & $d \in(-\infty, \infty) \backslash\{0\}$ & $\mathcal{E}_{x}(1)=1$ & $\mathcal{E}_{x}(0)=0$ \\
\hline$\varphi(x)=\exp \left(\frac{d}{x-1}\right)$ & $d \in[2, \infty)$ & $\mathcal{E}_{x}(1)=\infty$, & $\varphi(0)<\infty$ \\
\hline$\varphi(x)=\exp (d / x)-e^{d}$ & $d \in(0, \infty)$ & $\mathcal{E}_{x}(1)=1$ & $\mathcal{E}_{x}(0)=-\infty$ \\
\hline$\varphi(x)=\exp \left(x^{-d}\right)-e$ & $d \in(0, \infty)$ & $\mathcal{E}_{x}(1)=1$, & $\mathcal{E}_{x}(0)=-\infty$ \\
\hline$\varphi(x)=1-\left(1-(1-x)^{d}\right)^{1 / d}$ & $d \in[1, \infty)$ & $\mathcal{E}_{x}(1)=d$, & $\varphi(0)<\infty$ \\
\hline$\varphi(x)=\arcsin \left(1-x^{d}\right)$ & $d \in(0,1]$ & $\mathcal{E}_{x}(1)=1$, & $\varphi(0)<\infty$ \\
\hline
\end{tabular}

Note that the above assumptions are satisfied by all one-parameter families of archimedean copulas listed in [6, pp. 94-97]. Therefore all of them have uniform tail expansions. Below we list the corresponding 23 families of $\varphi$ 's, restrictions on $d$, and the values of $\mathcal{E}_{x}(1)$ and $\mathcal{E}_{x}(0)$ (when $\varphi(0)=\infty$ ). 
5.4. Examples. Let $\varphi(x)=(-\ln (x))^{d}$, where $d \in[1, \infty)$. Then

$$
\lim _{x \rightarrow 0} \mathcal{E}_{x} \varphi(x)=0, \quad \lim _{x \rightarrow 0} \mathcal{E}_{x} \varphi(1-x)=d .
$$

Hence the Gumbel-Hougaard copulas

$$
C(u, v)=\exp \left(-\sqrt[d]{(-\ln (u))^{d}+(-\ln (v))^{d}}\right)
$$

have the lower leading part equal to 0 and the upper one equal to

$$
u+v-\sqrt[d]{u^{d}+v^{d}}
$$

Similarly the BEV (bivariate extreme value) copulas

$$
C(u, v)=\exp (\ln (u)+\ln (v)+H(-\ln (u),-\ln (v))),
$$

if $H(x, y) \not \equiv \min (x, y)$, have the lower leading part equal to 0 and the upper one equal to $H(u, v)$.

In the exceptional case, $H(x, y)=\min (x, y), C(u, v)=\min (u, v)$. Hence in this case both leading forms equal $\min (u, v)$.

\section{Applications: Measuring the extreme risk of two long posi-} tions. We shall focus on the following simple case. An investor has in his portfolio two risky assets which are highly dependent. Let $\omega_{i}$ be the amount of money invested in the $i$ th asset. The investor fixes a security level $c$, $0<c<1$. In order to determine the risk associated with his investment he wants to estimate the following quantities:

- the probability that the value of the portfolio after a certain period of time is smaller than $c\left(\omega_{1}+\omega_{2}\right)$;

- the conditional probability that the value of one asset will be smaller than $c$ times its initial value, under the condition that this happens for the other asset;

- the conditional probability that the value of both assets will be smaller than $c$ times their initial values, under the condition that this happens for one of them;

- the conditional probability that the drop of the value of one asset will be greater than that of the other under the condition that the value of the other asset will be smaller than $c$ times its initial value.

Let $S_{i, 0}$ and $S_{i, 1}$ be the prices at the beginning and at the end of the period. So the final value of the investment equals

$$
W_{1}=\omega_{1} \frac{S_{1,1}}{S_{1,0}}+\omega_{2} \frac{S_{2,1}}{S_{2,0}} .
$$

We assume that the returns of both assets are heavy tailed, with the same tail index $\alpha>0$ (cf. [5, 9.3]), that is,

$$
\mathbb{P}\left(\ln \left(S_{i, 1}\right)-\ln \left(S_{i, 0}\right)<z\right) \approx a_{i}(-z)^{-\alpha} \quad \text { for } z \ll 0,
$$


and their joint distribution is determined by a copula $C$ having a uniform lower tail expansion with nonzero leading part $L$.

Let $c, 0<c<1$, be a benchmark, let

$$
F(c)=\mathbb{P}\left(W_{1}<c\left(\omega_{1}+\omega_{2}\right)\right),
$$

and let $U(c)$ and $V(c)$ be the probabilities that the value of respectively the first or second asset drops below $c$ :

$$
U(c)=\mathbb{P}\left(\frac{S_{1,1}}{S_{1,0}}<c\right), \quad V(c)=\mathbb{P}\left(\frac{S_{2,1}}{S_{2,0}}<c\right) .
$$

Obviously, for small $c$,

$$
U(c) \approx a_{1}(-\ln (c))^{-\alpha}, \quad V(c) \approx a_{2}(-\ln (c))^{-\alpha} .
$$

Lemma 4. For $\omega_{1}, \omega_{2}>0$ and sufficiently small $c$ we have the estimate

$$
F(c) \approx(-\ln (c))^{-\alpha} L\left(a_{1}, a_{2}\right) .
$$

Proof. To get the lower estimate, let $z=\ln c$. Then

$$
\begin{aligned}
F(c) & =\mathbb{P}\left(W \leq c\left(\omega_{1}+\omega_{2}\right)\right) \geq \mathbb{P}\left(\frac{S_{1,1}}{S_{1,0}} \leq c \wedge \frac{S_{2,1}}{S_{2,0}} \leq c\right) \\
& \approx C\left(a_{1}(-z)^{-\alpha}, a_{2}(-z)^{-\alpha}\right) \approx L\left(a_{1}(-z)^{-\alpha}, a_{2}(-z)^{-\alpha}\right) \\
& =L\left(a_{1}, a_{2}\right)(-z)^{-\alpha} .
\end{aligned}
$$

For the upper estimate, let $\omega=\ln \left(\frac{\min \left(\omega_{1}, \omega_{2}\right)}{\omega_{1}+\omega_{2}}\right)$ and $z<\omega$. Then

$$
\begin{aligned}
& \mathbb{P}\left(W \leq c\left(\omega_{1}+\omega_{2}\right)\right) \leq \mathbb{P}\left(\frac{S_{1,1}}{S_{1,0}} \leq c \frac{\omega_{1}+\omega_{2}}{\omega_{1}} \wedge \frac{S_{2,1}}{S_{2,0}} \leq c \frac{\omega_{1}+\omega_{2}}{\omega_{2}}\right) \\
\leq & \mathbb{P}\left(\frac{S_{1,1}}{S_{1,0}} \leq c \frac{\omega_{1}+\omega_{2}}{\min \left(\omega_{1}, \omega_{2}\right)} \wedge \frac{S_{2,1}}{S_{2,0}} \leq c \frac{\omega_{1}+\omega_{2}}{\min \left(\omega_{1}, \omega_{2}\right)}\right) \\
\approx & C\left(a_{1}(-z+\omega)^{-\alpha}, a_{2}(-z+\omega)^{-\alpha}\right) \approx L\left(a_{1}(-z+\omega)^{-\alpha}, a_{2}(-z+\omega)^{-\alpha}\right) \\
= & L\left(a_{1}, a_{2}\right)(-z+\omega)^{-\alpha} \approx L\left(a_{1}, a_{2}\right)(-z)^{-\alpha}\left(1-\alpha \frac{\omega}{(-z)}+\cdots\right) \\
\approx & L\left(a_{1}, a_{2}\right)(-z)^{-\alpha} .
\end{aligned}
$$

Note that since $L\left(a_{1}, a_{2}\right) \leq \min \left(a_{1}, a_{2}\right)$ we get:

Corollary 3. "Asymptotically", diversification of a portfolio does not increase the risk.

The answers to the next two questions from the above list follow from the classical formula for the conditional probability:

$$
\mathbb{P}\left(\frac{S_{1,1}}{S_{1,0}} \leq c \mid \frac{S_{2,1}}{S_{2,0}} \leq c\right)=\frac{\mathbb{P}\left(\frac{S_{1,1}}{S_{1,0}} \leq c \wedge \frac{S_{2,1}}{S_{2,0}} \leq c\right)}{\mathbb{P}\left(\frac{S_{2,1}}{S_{2,0}} \leq c\right)}=\frac{C(U(c), V(c))}{V(c)} .
$$


So for sufficiently small $c$,

$$
\mathbb{P}\left(\frac{S_{1,1}}{S_{1,0}} \leq c \mid \frac{S_{2,1}}{S_{2,0}} \leq c\right) \approx \frac{L(U(c), V(c))}{V(c)}=L\left(\frac{U(c)}{V(c)}, 1\right) \approx L\left(\frac{a_{1}}{a_{2}}, 1\right) .
$$

In the same way we show that

$$
\mathbb{P}\left(\frac{S_{2,1}}{S_{2,0}} \leq c \mid \frac{S_{1,1}}{S_{1,0}} \leq c\right) \approx \frac{L(U(c), V(c))}{U(c)}=L\left(1, \frac{V(c)}{U(c)}\right) \approx L\left(1, \frac{a_{2}}{a_{1}}\right) .
$$

The formula for the probability of two drops below the benchmark is only a bit more complicated:

$$
\begin{aligned}
\mathbb{P}_{2 \mid 1} & =\mathbb{P}\left(\frac{S_{1,1}}{S_{1,0}} \leq c \wedge \frac{S_{2,1}}{S_{2,0}} \leq c \mid \frac{S_{1,1}}{S_{1,0}} \leq c \vee \frac{S_{2,1}}{S_{2,0}} \leq c\right) \\
= & \frac{\mathbb{P}\left(\frac{S_{1,1}}{S_{1,0}} \leq c \wedge \frac{S_{2,1}}{S_{2,0}} \leq c\right)}{\mathbb{P}\left(\frac{S_{1,1}}{S_{1,0}} \leq c \vee \frac{S_{2,1}}{S_{2,0}} \leq c\right)}=\frac{C(U(c), V(c))}{U(c)+V(c)-C(U(c), V(c))}
\end{aligned}
$$

Therefore for sufficiently small $c$,

$$
\mathbb{P}_{2 \mid 1} \approx \frac{L(U(c), V(c))}{U(c)+V(c)-L(U(c), V(c))} \approx \frac{L\left(a_{1}, a_{2}\right)}{a_{1}+a_{2}-L\left(a_{1}, a_{2}\right)} .
$$

Corollary 4. For sufficiently small $c$ the above conditional probabilities do not depend on $c$.

The conditional probability that the drop of the value of the first asset will be greater than that of the second under the condition that the value of the second asset will be smaller than $c$ times its initial value equals (for differentiable $L(u, v)$ )

$$
\mathbb{P}_{1<2 \mid 2} \approx \frac{\partial L}{\partial v}\left(a_{1}, a_{2}\right)
$$

and the conditional probability that the drop of the value of the second asset will be greater than that of the first under the condition that the value of the first asset will be smaller than $c$ times its initial value equals

$$
\mathbb{P}_{2<1 \mid 1} \approx \frac{\partial L}{\partial u}\left(a_{1}, a_{2}\right)
$$

The following lemma is the crucial point of the estimation.

LEMMA 5. Let $\mu$ be a measure induced by $L(u, v)$. Then

$$
\begin{aligned}
& \mu_{1}=\mu\left(\triangle((0,0) ;(0, q) ;(p, q))=q \frac{\partial L}{\partial v}(p, q),\right. \\
& \mu_{2}=\mu\left(\triangle((0,0) ;(p, 0) ;(p, q))=p \frac{\partial L}{\partial u}(p, q) .\right.
\end{aligned}
$$




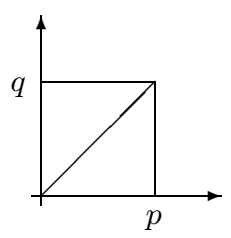

Proof. We have

$$
\mu_{1}=\int_{0}^{q} \int_{0}^{p v / q} \frac{\partial^{2} L}{\partial u \partial v}(u, v) d u d v=\int_{0}^{q} \frac{\partial L}{\partial v}\left(\frac{p v}{q}, v\right) d v
$$

Since $L$ is homogeneous of degree 1 , its first derivatives are homogeneous of degree 0 and

$$
\frac{\partial L}{\partial v}\left(\frac{p v}{q}, v\right)=\frac{\partial L}{\partial v}(p, q)
$$

Therefore

$$
\mu_{1}=\int_{0}^{q} \frac{\partial L}{\partial v}(p, q) d v=q \frac{\partial L}{\partial v}(p, q) .
$$

In the same way we get the formula for $\mu_{2}$.

Proof of approximations. By the lemma we have the following approximations:

$$
\begin{aligned}
& \mathbb{P}\left(\frac{S_{1,1}}{S_{1,0}} \leq \frac{S_{2,1}}{S_{2,0}} \leq c\right) \\
& =\mathbb{P}\left(a_{1} a_{2}\left(-\ln \left(\frac{S_{1,1}}{S_{1,0}}\right)\right)^{-\alpha} \leq a_{1} a_{2}\left(-\ln \left(\frac{S_{2,1}}{S_{2,0}}\right)\right)^{-\alpha} \leq a_{1} a_{2}(-\ln (c))^{-\alpha}\right) \\
& \approx \mathbb{P}\left(a_{2} U\left(\frac{S_{1,1}}{S_{1,0}}\right) \leq a_{1} V\left(\frac{S_{2,1}}{S_{2,0}}\right) \leq a_{1} a_{2}(-\ln (c))^{-\alpha}\right) \\
& \approx \mu\left(\triangle\left((0,0) ;\left(0, a_{2}(-\ln (c))^{-\alpha}\right) ;\left(a_{1}(-\ln (c))^{-\alpha}, a_{2}(-\ln (c))^{-\alpha}\right)\right)\right. \\
& =a_{2}(-\ln (c))^{-\alpha} \frac{\partial L}{\partial v}\left(a_{1}(-\ln (c))^{-\alpha}, a_{2}(-\ln (c))^{-\alpha}\right) \\
& =a_{2}(-\ln (c))^{-\alpha} \frac{\partial L}{\partial v}\left(a_{1}, a_{2}\right) .
\end{aligned}
$$

Therefore

$$
\begin{aligned}
\mathbb{P}_{1<2 \mid 2} & =\mathbb{P}\left(\frac{S_{1,1}}{S_{1,0}} \leq \frac{S_{2,1}}{S_{2,0}} \mid \frac{S_{2,1}}{S_{2,0}} \leq c\right)=\frac{\mathbb{P}\left(\frac{S_{1,1}}{S_{1,0}} \leq \frac{S_{2,1}}{S_{2,0}} \leq c\right)}{\mathbb{P}\left(\frac{S_{2,1}}{S_{2,0}} \leq c\right)} \\
& \approx \frac{a_{2}(-\ln (c))^{-\alpha} \frac{\partial L}{\partial v}\left(a_{1}, a_{2}\right)}{a_{2}(-\ln (c))^{-\alpha}}=\frac{\partial L}{\partial v}\left(a_{1}, a_{2}\right) .
\end{aligned}
$$

In the same way we get the approximation of $\mathbb{P}_{2<1 \mid 1}$. 


\section{References}

[1] P. Embrechts, L. de Haan and X. Huang, Modelling multivariate extremes, in: P. Embrechts (ed.), Extremes and Integrated Risk Management, Risk Waters Group Ltd, 2000, 59-67.

[2] W. Hürlimann, Hutchinson-Lai's conjecture for bivariate extreme value copulas, Statist. Probab. Lett. 61 (2003), 191-198.

[3] J. Jakubowski and R. Sztencel, Introduction to Probability Theory, SCRIPT, Warszawa, 2000 (in Polish).

[4] P. Jaworski, Asymptotics of bivariate copulas, Mat. Stos. 4 (2003), 78-89 (in Polish).

[5] R. N. Mantegna and H. E. Stanley, An Introduction to Econophysics. Correlations and Complexity in Finance, Cambridge Univ. Press, 2000.

[6] R. B. Nelsen, An Introduction to Copulas, Springer, 1999.

[7] R. T. Rockafellar, Convex Analysis, Princeton Univ. Press, Princeton, 1970.

Institute of Mathematics

Warsaw University

Banacha 2

02-097 Warszawa, Poland

E-mail: jwptxa@mimuw.edu.pl

Received on 12.1.2004;

revised version on 7.9.2004 